Copyright by the Acoustical Society of America. Maillard, J. P. \& Fuller, C. R. (1998). Comparison of two structural sensing approaches for active structural acoustic control. Journal of the Acoustical Society of America, 103(1), 396-400. doi: 10.1121/1.421112

\title{
Comparison of two structural sensing approaches for active structural acoustic control
}

\author{
Julien P. Maillard and Chris R. Fuller \\ Vibration and Acoustics Laboratories, Mechanical Engineering Department, Virginia Polytechnic Institute \\ and State University, Blacksburg, Virginia 24061-0238
}

(Received 23 December 1996; accepted for publication 7 July 1997)

\begin{abstract}
A numerical study comparing the use of two structural sensing approaches for sound radiation control is performed on a baffled rectangular plate. The first sensing approach implements an array of accelerometers whose outputs are filtered to construct an estimate of the sound pressure radiated at given angles in the far field. The second method uses the same array of point sensors to estimate the net volume acceleration of the plate. Results show the improved performances of the sensor observing far-field sound radiation over a volume acceleration based on sensor. (C) 1998 Acoustical Society of America. [S0001-4966(98)00801-7]
\end{abstract}

PACS numbers: 43.40.At, 43.40.Vn, 43.40.Rj [PJR]

\section{INTRODUCTION}

An important issue in the active control of structurally radiated noise remains the design and implementation of error sensors. The early works on active structural acoustic control (ASAC) ${ }^{1}$ were concentrated on the design of structural actuators ${ }^{2}$ while the error signals to minimize were provided by microphones located in the far field. For the last couple of years, the research in ASAC has made some progress in replacing error microphones by error sensors integrated in the structure. ${ }^{3}$ Unlike microphones which directly measure the quantity to be minimized, i.e., acoustic pressure, structural sensors only measure information related to the structural vibrations. The goal is then to design a structural sensor whose output is strongly related to radiated sound pressure so that minimizing the magnitude of the sensor output signal will also minimize the total radiated sound power. In other words, an efficient structural sensing technique should take into consideration the structure-fluid interaction such that only the radiating part of the structural vibrations is observed by the sensor. Indeed, minimizing both radiating and nonradiating components of the vibrations typically requires more control effort below the coincidence frequency.

Spatially distributed structural sensors such as polyvinylidene fluoride (PVDF) materials have shown potential for observing the radiating part of the structural vibrations. In modal sensing, the PVDF film is designed to observe the dominant radiating modes among those found in the structural response. ${ }^{4-6}$ The concept of "radiation modes" has also been used in designing error sensors. ${ }^{7,8}$ In particular, several authors have demonstrated the use of PVDF sensors designed to observe the first radiation mode of a rectangular plate. $^{8-11}$ This mode, also referred to as the piston mode, presents the greatest radiation efficiency of all radiation modes. It can be detected by measuring the net volume displacement over the surface of the structure and specially designed PVDF sensors have been constructed for this task. ${ }^{8-11}$

Recently, a sensing technique referred to as discrete structural acoustic sensing (DSAS) was demonstrated both analytically and experimentally on baffled planar radiators. ${ }^{12-14}$ The technique implements an array of struc- tural point sensors (usually accelerometers) whose outputs are passed through digital filters to estimate in real time the far-field radiated pressure in a given direction, or equivalently, a given wave-number component, over a broad frequency range. The digital filters are usually finite impulse response (FIR) filters designed to model the appropriate Green's function associated with each point sensor and farfield locations. It was shown that only a low number of point sensors is needed to provide accurate sound radiation estimates over a bandwidth encompassing the first few modes of a rectangular plate. Moreover, the sensor's output can be shifted in time such that the acoustic path delay present in the Green's functions is removed. This allows the use of only a few coefficients in the sensor FIR filter array and makes its implementation on a digital signal processor (DSP) very efficient in terms of computational load.

A simplified version of the above approach that has been suggested consists of replacing the radiation Green's functions by a unity transfer function. The sensor output then simply becomes the sum of the acceleration signals. The resulting error information thus represents an estimate of the net volume acceleration of the structure. It is therefore somewhat equivalent to the PVDF volume velocity sensor referred above except it is implemented in discrete form. This alternative sensing approach will be referred to as discrete structural volume acceleration sensing (DSVAS). For planar radiators, this error information is also equivalent to measuring the far-field pressure in the direction normal to the plane of the radiator. ${ }^{15}$

This paper presents a short numerical study comparing the performance of the two sensing approaches introduced above, i.e., DSAS and DSVAS methods, in active structural acoustic control applied to a rectangular baffled plate. The plate is excited by a normal point force over a bandwidth encompassing its first nine flexural modes. The control is achieved through two single-sided piezoelectric patches. The system modeling is briefly described in the first two sections along with the plate's physical characteristics. The third section then presents the control performance in terms of mean- 
square velocity and radiated power attenuation obtained for various sensing configurations.

\section{SYSTEM MODELING}

The numerical model describing the structural response of the plate excited by point forces and piezoelectric patches is briefly presented. In this model, the mechanical displacements and electrical fields generated in the piezoelectric inclusions are fully coupled. In order to solve for the dynamics of this coupled electromechanical system, a Rayleigh-Ritz formulation is derived based on the generalized form of Hamilton's principle for coupled electromechanical system. ${ }^{16}$ This energy-based method also allows modeling of arbitrary boundary conditions applied along the edges of the plate. The plate midplane displacements along the two longitudinal and transverse directions are approximated by a polynomial series where the unknown polynomial amplitudes are the solution of a linear system. After solving this system at each frequency of interest, various structural quantities, such as mean-square velocity, or point acceleration, can be computed. For a complete derivation of this approach applied to rectangular plates, the reader is referred to the work of Berry. ${ }^{17}$

The far-field sound pressure radiated from the plate is obtained from the normal displacement amplitudes by computing the continuous wave-number transform of the acceleration distribution over the frequency bandwidth. The radiated power is estimated from the radiation impedance matrix associated with the polynomial basis used to approximate the structural response. This approach is more efficient than simply integrating the far-field pressure over the hemisphere surrounding the plate. More details can be found in Ref. 18 .

The output from the discrete structural acoustic sensor is computed from the structural acceleration evaluated at nine point sensor locations on the plate and multiplied by the appropriate sensor transfer functions as described in Ref. 14. Note that the sensor transfer functions used in this numerical study correspond to the ideal case of perfect filter modeling. It was shown in previous work, however, that these transfer functions are easily modeled to a high level of accuracy with only a few coefficients per FIR filter. ${ }^{12}$

The controlled structural response is obtained by adding to the uncontrolled response due to the point force excitation, the control field response due to the two one-sided piezoelectric patches. The optimal control voltage to each PZT is computed using standard linear quadratic optimal control theory (see, for example, Ref. 19), where the cost function to be minimized is a quadratic function of the control voltage amplitudes. It should be noted that this frequency domain optimal controller does not take into account the causality constraint inherent to a real-time implementation on a DSP. In other words, the optimal controller transfer functions in the frequency domain might be acausal and therefore impossible to reproduce using a digital filter. This results in overestimating the attenuation achieved by the controller, however, it does represent the maximum achievable attenuation if the system is causal. Also, the limitation due to the finite dynamic range of the analog to digital converters is not included in this model.

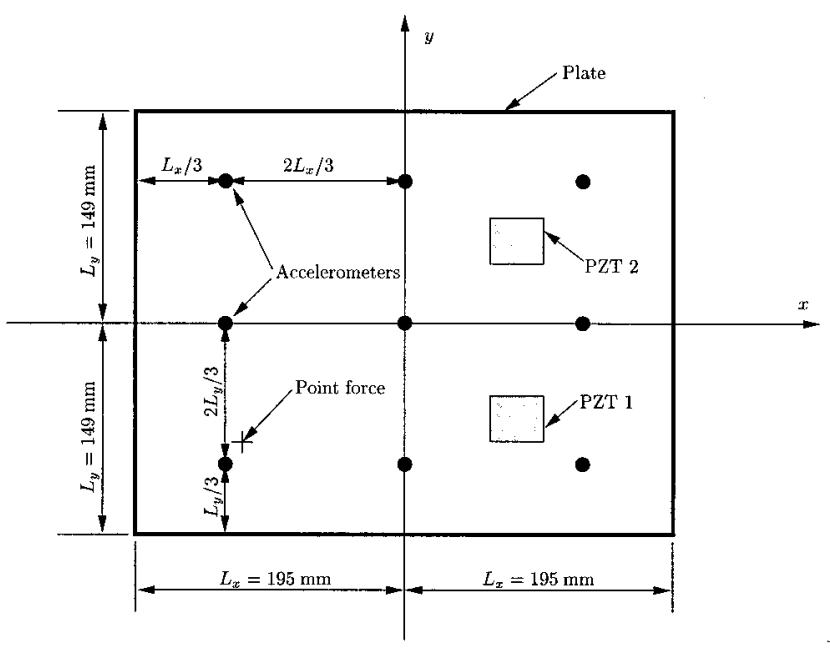

FIG. 1. Plate geometry and transducer locations.

As mentioned above, the plate is excited by a point force while two PZT patches are implemented to achieve control. Preliminary tests showed that a single piezoelectric actuator was not sufficient to achieve global sound radiation attenuation over the entire frequency bandwidth. The sensor based on unit transfer functions, i.e., estimating the structural net volume acceleration, provides one single error signal. To facilitate the comparison with the sensor based on far-field pressure estimates, only one far-field direction is considered. This control layout, i.e., two control outputs and one error input, results in an underdetermined system which is solved by including in the cost function the weighted control effort (sum of the square modulus of each control voltage) as an additional quantity to minimize, effectively constraining the solution. This second variable is scaled down (by varying its weighting) so that the main forces of the control are directing towards minimizing the error information rather than the control voltages.

\section{SYSTEM CHARACTERISTICS}

The system studied here is a model based on the plate tested experimentally in a previous paper. ${ }^{14}$ The translation and rotation stiffness factors along each side of the plate were numerically adjusted in an effort to match the experimental natural frequencies. The resulting modeled boundary conditions were close to the simply supported case. The plate geometry is shown in Fig. 1 along with the location of the normal point force disturbance, the two PZT control inputs, and the nine accelerometers implemented in the discrete structural acoustic sensor. The physical parameters and dimensions for the plate and piezoelectric patches are shown in Table I. The point force and PZT center locations are presented in Table II. As mentioned earlier, the PZT patches are single-sided. The impact of the resulting asymmetry will be discussed later in the paper. The control actuator locations in Table II were optimally chosen for minimizing the $(3,1)$ and $(1,3)$ flexural modes, while the disturbance force location ensures all nine modes present in the bandwidth of interest are excited. Finally, the model's natural frequencies are shown in Table III. 
TABLE I. Plate and PZT's dimensions and material properties.

\begin{tabular}{lll}
\hline \hline Parameter & Plate & PZT \\
\hline Length in $x$ axis $(\mathrm{mm})$ & 380 & 38 \\
Length in $y$ axis $(\mathrm{mm})$ & 298 & 32 \\
Thickness, $h(\mathrm{~mm})$ & 1.93 & 0.1905 \\
Young's modulus $\left(\mathrm{N} / \mathrm{m}^{2}\right)$ & $2.04 \times 10^{11}$ & $6.1 \times 10^{10}$ \\
Poisson ratio & 0.28 & 0.33 \\
Mass density $\left(\mathrm{kg} / \mathrm{m}^{3}\right)$ & 7700 & 7750 \\
Hysteretic damping factor & 0.0005 & 0 \\
$d_{31}$ constant $(\mathrm{m} / \mathrm{V})$ & $\ldots$ & $171 \times 10^{-12}$ \\
\hline \hline
\end{tabular}

The structure's response is computed over the frequency bandwidth, 50-650 Hz, with a $1-\mathrm{Hz}$ increment. Note that the peak levels of the response at those frequencies is likely to be significantly below the actual resonance level reached at the resonance frequency: The small level of damping included in the system (see Table I) results in sharp resonance peaks relative to the above frequency resolution.

\section{CONTROL PERFORMANCES}

Figure 2 shows the sound radiated power plotted versus frequency for the uncontrolled plate (solid line) and the controlled plate using the two sensing approaches described earlier. The dashed line corresponds to the DSAS approach (pressure estimate based sensor) and the dotted line to the DSVAS approach (net volume acceleration based sensor). The direction of minimization for the DSAS approach is set to $\left(\theta=36^{\circ}, \phi=30^{\circ}\right)$. This direction was chosen so that it does not coincide with any of the nodal lines of the radiation directivity of the modes present in the bandwidth. In other words, the sensor based on the far-field pressure estimate observes the radiation of all the flexural modes of the plate.

The first sensing approach of DSAS achieves good control over the entire bandwidth with a very small amount of spillover. The largest level of attenuation is obtained for mode $(1,1)$ around $86 \mathrm{~Hz}$. Very good attenuation is also seen for the other modes present in the frequency bandwidth except for the resonance at $333 \mathrm{~Hz}$ corresponding to the $(2,2)$ mode of the plate. Around this frequency, the control system only reduces the radiated power level by about $10 \mathrm{~dB}$. Looking at the pressure spatial directivity of the uncontrolled and controlled plate at this frequency reveals that the pressure distribution is slightly shifted along the $\phi$ direction so that the $(2,2)$ mode radiation nodal lines go through the direction of minimization $\left(\theta=36^{\circ}, \phi=30^{\circ}\right)$. In other words, the amplitude of this mode is not reduced. Indeed, the structural velocity distribution at this frequency shows hardly any changes between the uncontrolled and controlled responses. This phenomenon is commonly referred to as modal restructuring. This is a direct consequence of minimizing the sound-

TABLE II. Transducer location.

\begin{tabular}{lcc}
\hline \hline \multicolumn{1}{c}{ Transducer type } & Center coordinate, $x / L_{x}$ & Center coordinate, $y / L_{y}$ \\
\hline Disturbance point force & -0.60526 & -0.56376 \\
Control PZT 1 & 0.41579 & -0.45302 \\
Control PZT 2 & 0.41579 & 0.38926 \\
\hline \hline
\end{tabular}

TABLE III. Plate natural frequencies.

\begin{tabular}{cc}
\hline \hline Mode $(m, n)$ & Natural frequency $(\mathrm{Hz})$ \\
\hline$(1,1)$ & 86.0 \\
$(2,1)$ & 181.2 \\
$(1,2)$ & 241.4 \\
$(2,2)$ & 332.7 \\
$(3,1)$ & 339.1 \\
$(3,2)$ & 484.6 \\
$(1,3)$ & 494.5 \\
$(4,1)$ & 557.7 \\
$(2,3)$ & 580.7 \\
$(4,2)$ & 695.5 \\
\hline \hline
\end{tabular}

pressure field in a single direction. To obtain a larger level of sound power reduction, additional far-field pressure error estimates in other directions could be included in the control system. Or alternatively, a single error signal directly related to the radiated power would also provide better overall sound attenuation.

The latter solution motivates the use of a sensor based on net structural volume acceleration. The resulting error information directly relates to the volumetric modes of the radiators, i.e., the odd-odd modes for a plate. These modes are the most efficient radiators, and the sensor output is therefore directly related to sound radiated power. This approach corresponds to the dotted line where the sensor output is the sum of the nine acceleration signals (unit transfer function). As seen in Fig. 2, this second sensing approach performs well near the resonance frequencies of the odd-odd modes of the plate. On the other hand, the radiated power around the resonances of odd-even or even-even modes is not significantly reduced. The global radiation level of the $(2,1)$ mode, for example, even increases after control. By definition, the net volume acceleration-based sensor does not observe any of the odd-even or even-even modes of the plate. Therefore the system is unable to effectively control these modes. However, they still contribute to the total radiated power, despite their lower radiation efficiency compared to the odd-odd modes. In fact for some frequencies they can radiate more power than volumetric modes. ${ }^{3}$

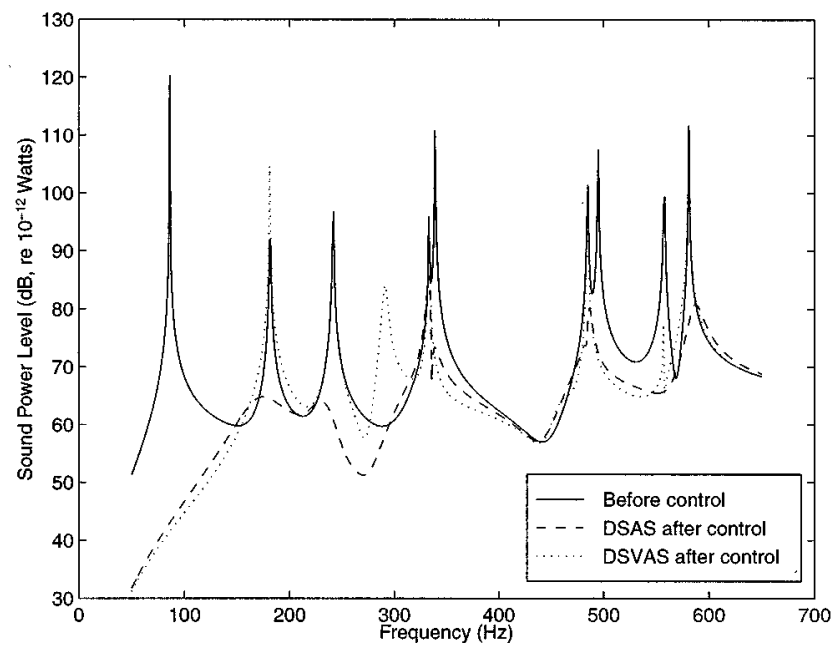

FIG. 2. Radiated power. 


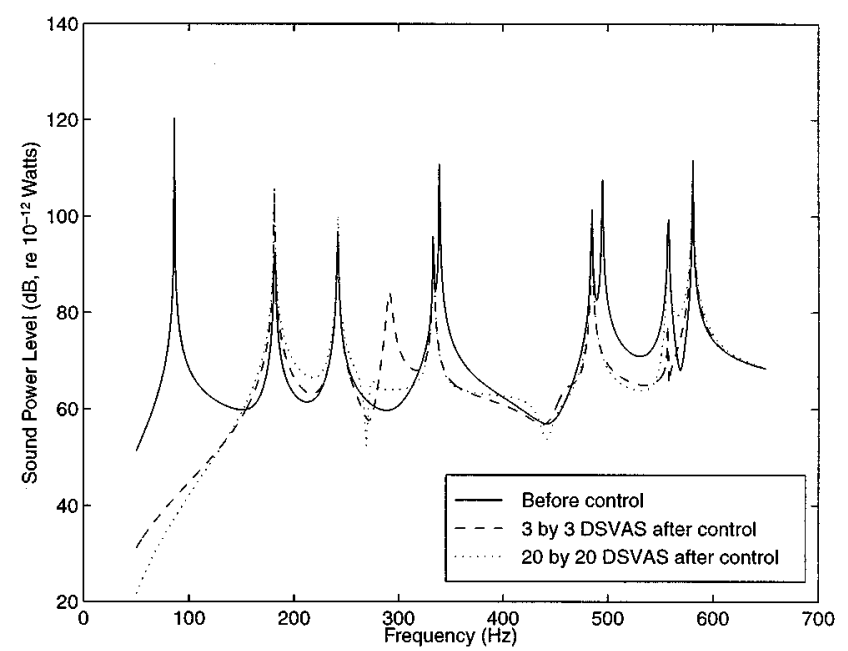

FIG. 3. Radiated power.

Another interesting feature of the control performance of the volume acceleration based sensor is the large control spillover occurring around $290 \mathrm{~Hz}$. In this frequency range, the controlled structural response exhibits a velocity distribution which perfectly cancels the summation of the nine acceleration amplitudes. However, this velocity distribution radiates significantly in the far field which explains the control spillover. Examining the radiated power level at the resonance frequency of mode $(4,1)$, significant reduction can be observed (dotted line) even though the symmetry of the velocity distribution associated with the $(4,1)$ mode should cancel the sensor output. This phenomenon is explained by the presence of the two PZT patches in the model resulting in slightly asymmetric modes. These slight differences in magnitude and phase of the acceleration amplitudes measured at the nine locations shown in Fig. 1 result in a nonzero error signal amplitude. Note that these differences are averaged out when using a higher level of discretization. To illustrate this point, another simulation was performed where the sensor uses a $20 \times 20$ grid of acceleration point measurements to estimate the net volume acceleration. This level of discretization ensures an almost perfect estimate of the net volume acceleration in the frequency bandwidth of interest. Figure 3 shows the radiated power before (solid line) and after control using the previous $3 \times 3$ sensor (dashed line) and the 20 $\times 20$ sensor (dotted line). The $20 \times 20$ sensor yields a much more accurate estimate of the net volume displacement. This is apparent around $558 \mathrm{~Hz}$ where the $(4,1)$ mode is not observed by the sensor and results in no radiated power attenuation. Also, the system's behavior noticed in the case of the $3 \times 3$ sensor around $290 \mathrm{~Hz}$ no longer appears with the 20 $\times 20$ sensor. In conclusion, even though the $3 \times 3$ sensor might yield larger global attenuation in some cases such as at a frequency around $558 \mathrm{~Hz}$, a higher level of discretization ensures a better estimate of the net volume acceleration and thus enables the controller to observe all volumetric velocity distributions. Note that a PVDF continuous sensor designed to observe the same information is therefore likely to perform better compared to a $3 \times 3$ sensor. However, several authors have shown the difficulties of designing such a sen-
TABLE IV. Total mean-square velocity and radiated power attenuation.

\begin{tabular}{lcc}
\hline \hline Attenuation $(\mathrm{dB})$ & Mean-square velocity & Radiated power \\
\hline Error mic. & 17.7 & 23.8 \\
DSAS & 17.1 & 23.8 \\
DSVAS $(3 \times 3)$ & 5.5 & 10.1 \\
DSVAS $(20 \times 20)$ & 3.9 & 8.2 \\
\hline \hline
\end{tabular}

sor with the required accuracy, especially for twodimensional radiators. ${ }^{9,11}$

To summarize the above results, the reduction levels for the total mean-square velocity and sound radiated power computed over the frequencies of interest are shown in Table IV. The first line corresponds to an additional simulation case where the far-field pressure error estimate (sensor output) is replaced by the actual far-field pressure evaluated in the same direction. Note that this configuration nearly yields the same level of reductions as the pressure estimate structural sensor (DSAS). This demonstrates the ability of the sensor to replace far-field measurements as it was shown in previous work. On the other hand, the sensors based on the net volume acceleration (DSVAS) yield a small level of overall reduction $(10 \mathrm{~dB}$ for the $3 \times 3$ sensor and $8 \mathrm{~dB}$ for the $20 \times 20$ sensor) when compared to the performance of the DSAS sensor. The higher levels of attenuation achieved by the $3 \times 3$ sensor are consistent with the curves of Fig. 3 discussed earlier. Despite strong attenuation levels at the resonance frequencies of the odd-odd modes, the overall reduction level remains small due to the remaining peaks in the controlled response of the odd-even and even-even modes.

\section{CONCLUSIONS}

The discrete structural volume acceleration sensing (DSVAS) approach, i.e., based on unit transfer functions, performs well in the low-frequency range below the resonance of the first nonvolumetric mode of the plate. Due to its simplicity, it is the recommended method in this frequency range. At higher frequencies, the discrete structural acoustic sensing (DSAS) approach, i.e., based on radiation transfer functions, provides much increased performance due to the fact that it observes radiation from all modes. Thus near and above the resonance frequency of the first nonvolumetric mode, DSAS is the recommended sensing approach for ASAC.

\section{ACKNOWLEDGMENTS}

The financial support of the Office of Naval Research, Dr. Kam Ng, Technical Monitor for this work is gratefully acknowledged.

${ }^{1}$ C. R. Fuller, "Active control of sound transmission/radiation from elastic plates by vibration inputs. I. Analysis," J. Sound Vib. 136, 1-15 (1990).

${ }^{2}$ C. R. Fuller, C. H. Hansen, and S. D. Snyder, "Active control of structurally radiated noise using piezoceramic actuators," in Proceedings of Inter-Noise 89, Newport Beach, CA, edited by G. C. Maling (Noise Control Foundation, Poughkeepsie, NY, 1989), pp. 509-511.

${ }^{3}$ C. R. Fuller, S. J. Elliott, and P. A. Nelson, Active Control of Vibration (Academic, London, 1996).

${ }^{4}$ C. K. Lee and F. C. Moon, "Modal sensors/actuators," Trans. ASME, J. Appl. Mech. 57, 434-441 (1990). 
${ }^{5}$ Yi Gu, R. L. Clark, C. R. Fuller, and A. C. Zander, "Experiments on active control of plate vibration using piezoelectric actuators and polyvinylidene (PVDF) modal sensors,"' ASME J. Vib. Acoust. 116, 303-308 (1994).

${ }^{6}$ R. L. Clark and C. R. Fuller, "Modal sensing of efficient radiators with PVDF distributed sensors in active structural acoustic approaches," J. Acoust. Soc. Am. 91, 3321-3329 (1992).

${ }^{7}$ G. V. Borgiotti, "The power radiated by a vibrating body in an acoustic fluid and its determination from boundary measurements," J. Acoust. Soc. Am. 88, 1884-1893 (1990).

${ }^{8}$ M. E. Johnson and S. J. Elliott, "Volume velocity sensors for active control,' Proc. Inst. Acoust. 15, 411-420 (1993).

${ }^{9}$ J. Rex and S. J. Elliott, "'The QWSIS, a new sensor for structural radiation control,"' in First International Conference on Motion and Vibration Control, Yokohama (1992), pp. 339-343.

${ }^{10} \mathrm{C}$. Guigou, F. Charette, and A. Berry, "Active control of sound by minimization of volume velocity on finite beam," in Proceedings of the Third International Congress on Air- and Structure-Borne Sound and Vibration, Montreal, edited by M. J. Crocker (ME Dept. Auburn University, Auburn, AL, 1994), pp. 1507-1514.

${ }^{11}$ C. Guigou, A. Berry, and F. Charette, "Active control of plate volume velocity using shaped PVDF sensor," Adaptive Structures and Composite Materials: Analysis and Application ASME 45, 247-255 (1994).

${ }^{12}$ J. P. Maillard and C. R. Fuller, "Advanced time domain wave-number sensing for structural acoustic systems. I. Theory and design,' J. Acoust. Soc. Am. 95, 3252-3261 (1994).

${ }^{13}$ J. P. Maillard and C. R. Fuller, "Advanced time domain wave-number sensing for structural acoustic systems. II. Active radiation control of a simply-supported beam," J. Acoust. Soc. Am. 95, 3262-3272 (1994).

${ }^{14}$ J. P. Maillard and C. R. Fuller, "Advanced time domain wave-number sensing for structural acoustic systems. III. Experiments on active broadband radiation control of a simply-supported plate,' J. Acoust. Soc. Am. 98, 2613-2621 (1995).

${ }^{15}$ C. Guigou, Z. Li, and C. R. Fuller, "The relationship between volume velocity and far-field radiated pressure of a planar structure,' J. Sound Vib. 197, 252-254 (1996).

${ }^{16}$ N. W. Hagood, W. H. Chung, and A. Von Flotow, "Modeling of piezoelectric actuator dynamics for active structural control," AIAA J. 90(1087-CP), 2242-2256 (1990).

${ }^{17}$ A. Berry, J.-L. Guyader, and J. Nicolas, "A general formulation for the sound radiation from rectangular, baffled plates with arbitrary boundary conditions," J. Acoust. Soc. Am. 88, 2792-2802 (1990).

${ }^{18} \mathrm{~A}$. Berry, "Vibrations et rayonnement acoustique de structures planes complexes immergées dans un fluide léger ou dans un fluide lourd," $\mathrm{Ph} . \mathrm{D}$. thesis, Université de Sherbrooke, Sherbrooke, Canada, 1991.

${ }^{19}$ P. A. Nelson and S. J. Elliott, Active Control of Sound (Academic, London, 1992), pp. 416-420. 